\title{
Editorial for rare metals, special issue on advanced permanent magnetic materials
}

\author{
Ming Yue*(1), Jin-Bo Yang
}

Published online: 5 December 2019

(C) The Nonferrous Metals Society of China and Springer-Verlag GmbH Germany, part of Springer Nature 2019

Permanent magnetic materials (PMMs) are a kind of indispensable functional materials in modern society, which play crucial role in many applications such as computers, medical devices, and home appliances. Specially, with the rapid development of green technologies such as electric transportation, wind turbines, and precision motors, the need for high-quality PMMs has become strong and urgent. Meanwhile, the cutting-edge researches involving development and improvement of PMMs and related theories have drawn considerable attentions. This motives rare metals to have a special issue focusing on PMMs.

This special issue publishes 2 reviews and 11 research articles on both experimental and theoretical sides. The articles in this issue are selected from the proceedings of 2018 Rare Earth Permanent Magnets and Advanced Magnetic Materials and Their Applications (REPM 2018), which is a premier conference on all aspects of PMMs and reviews the latest developments in PMMs. The articles in this issue are from the research group from China, Korea, and Russia. The topic covers rare-earth-based magnets, transition-metal-based magnets, ferrite magnets, and micromagnetic simulation. We sincerely expect that the readers may find the articles published in this special issue informative and valuable.

\section{Yue*}

College of Materials Science and Engineering, Beijing University of Technology, Beijing 100124, China

e-mail: yueming@bjut.edu.cn

J.-B. Yang

School of Physics, Peking University, Beijing 100871, China

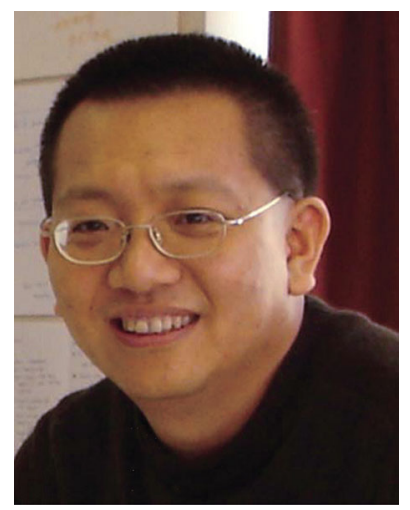

Ming Yue obtained his Ph.D. degree in Materials Science in 2001 from the University of Science and Technology Beijing. In 2009 and 2012, he respectively became a faculty professor and a doctor supervisor in the College of Materials Science and Engineering, Beijing University of Technology. His research interests involve high-performance permanent magnets. He has more than 50 patents and has published more than 200 technical articles in peer-reviewed science and engineering journals. $\mathrm{He}$ is an international editorial board member of two SCI-indexed journals: Rare Metals (China) and Journal of Magnetics (Korea), and an International Advisory Committee member of the Workshop on rare-earth permanent magnets.

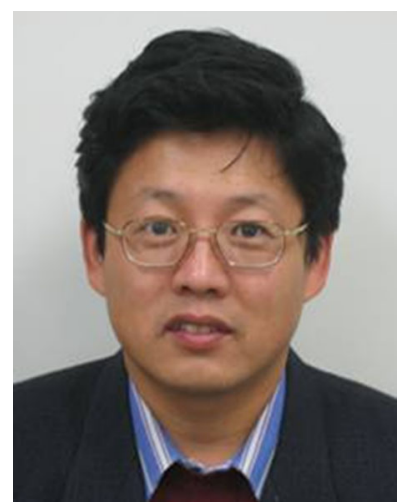

Jin-Bo Yang Professor of Physic, Peking University. Dr. Yang received his Ph.D. in Condensed Matter Physics from the Peking University (PKU) in 1998. He received the Alexander von Humboldt Fellowship and performed a post-doctor at IFWDresden Germany from 1998 to 2000. He then worked in University of Missouri-Rolla (UMR) from 2001 to 2008 . He became a full Professor in PKU in 2008. Prof. Yang is currently the member of the International Rare Earth Permanent Magnetic Materials Advisory Committee, Member of the International IEEE Magnetic Technology Committee, etc. His research works focus on the magnetic materials, magnetism, and spintronics involving the relationship between structure and physical properties of the novel magnetic functional materials, neutron scattering and magnetism, novel permanent and soft magnetic materials. He has published more than 250 peer-reviewed SCI papers and authorized more than 10 patents. He has received National Natural Science Award (second prize), the national 100 excellent doctoral thesis awards, and others. 